\title{
Droit comparé pour traducteurs : de la théorie à la didactique de la traduction juridique
}

\author{
Valérie Dullion
}

Published online: 31 January 2014

(C) Springer Science+Business Media Dordrecht 2014

\begin{abstract}
Theorists of legal translation generally describe it as an interdisciplinary activity whose methodology draws deeply upon comparative law. In practice, how can we apply this theoretical paradigm to translator training? This article examines methods of integrating comparative law with the acquisition of knowledge and know-how that constitute the translator's core competences, emphasizing the resolution of legal terminology problems resulting from incongruencies between legal systems. Given that the goal is to compare law for the purposes of translation, it is useful to approach these problems from a functionalist viewpoint, principally through confronting a range of cases and situations likely to challenge the professional translator. Therefore, the author proposes including, along with other teaching methods, targeted work on terminological problems which necessitate the study of comparative law. A set of learning activities is presented for that purpose.
\end{abstract}

Keywords Legal translation - Comparative law · Legal terminology · Competence $\cdot$ Translation didactics

Résumé Les théoriciens de la traduction juridique s'accordent pour présenter celle-ci comme une activité interdisciplinaire dont la méthodologie fait largement appel au droit comparé. Comment concrétiser cet « acquis théorique » sur le terrain de la formation des traducteurs ? Le présent article traite des moyens d'intégrer le droit comparé dans l'acquisition des savoirs et des savoir-faire qui constituent les compétences du traducteur. Il met l'accent sur la résolution des problèmes de terminologie juridique qui résultent de la divergence des notions entre systèmes.

\footnotetext{
V. Dullion $(\bowtie)$

Faculté de traduction et d'interprétation, Université de Genève, Uni Mail, 40, bd du Pont-d'Arve, 1211 Genève 4, Suisse e-mail: Valerie.Dullion@unige.ch
} 
Puisqu'il s'agit d'apprendre à comparer les droits pour traduire, il est utile d'aborder ces problèmes dans une optique fonctionnaliste, principalement à travers la confrontation de divers cas de figure et situations susceptibles de se présenter au traducteur professionnel. Dans cette perspective, l'auteur propose de faire une place, parmi les méthodes d'enseignement, à un travail ciblé sur les problèmes de terminologie nécessitant un passage par le droit comparé. Un ensemble d'activités didactiques sont présentées à cet effet.

Mots clés Traduction juridique · Droit comparé · Terminologie juridique · Compétence - Didactique de la traduction

\section{Introduction}

Définies dans le contexte de chaque société, les notions juridiques sont différentes d'une culture à l'autre. C'est pourquoi la traduction juridique passe, en général, par le droit comparé : tel est l'un des grands principes dégagés par la réflexion théorique qui la prend pour objet (voir p. ex. [6, pp. 12-15; 8, pp. 53-82 ; 16, pp. 145-147 ; 23 , pp. 105-122; 35, pp. 13-14 et 229-269]). Cette caractéristique de la traduction juridique contribue pour beaucoup à sa difficulté mais aussi à son intérêt, en la distinguant d'autres types de traduction spécialisée [36, p. 83], notamment de la traduction technique.

Si l'on considère que les problèmes de méthode propres à la traduction juridique en font une discipline exigeant une formation particulière, le droit comparé doit occuper dans cette formation une place de choix. Or les liens entre droit comparé et traduction juridique sont complexes et peuvent être abordés sous différentes perspectives [24]. De quel droit comparé les traducteurs ont-ils besoin et comment le leur enseigner?

Le présent article vise à répondre à ces questions en puisant, d'une part, dans les travaux de plusieurs disciplines et, d'autre part, dans une expérience de l'enseignement qui a permis d'élaborer des activités spécifiques et d'analyser les difficultés rencontrées au cours de l'apprentissage. Après avoir exposé les fondements juridiques, traductologiques et didactiques de notre réflexion (Sect. 2), nous montrerons quels éléments de théorie et de méthodologie les traducteurs peuvent emprunter au droit comparé (Sect. 3) et comment ils peuvent apprendre à se servir de ces outils pour la traduction (Sect. 4). Nous présenterons ensuite, à titre d'illustration, un ensemble d'activités susceptibles d'être intégrées dans l'enseignement (Sect. 5).

Notre propos se situe donc au point d'articulation entre théorie et didactique de la traduction (voir la démarche de Colina [9, p. 31]), dans un domaine de spécialité. Plus précisément, nous nous concentrons sur un aspect essentiel du traitement didactique de l'interdisciplinarité en traduction juridique. 


\section{Des compétences spécifiques, un travail ciblé}

\subsection{Le « casse-tête » de la terminologie juridique}

L'absence de symétrie entre deux systèmes juridiques peut se répercuter sur la traduction à tous les niveaux, des genres textuels (avec les conventions discursives qui les caractérisent) aux termes [1, pp. 20-22]. Les problèmes posés par ces derniers sont une préoccupation constante. Selon la formule de De Groot [11, p. 287], la traduction de la terminologie juridique est essentiellement une opération de droit comparé ${ }^{1}$. S'il est vrai que le domaine juridique n'a pas l'exclusivité des divergences notionnelles entre langues - ni des types de solutions que les traducteurs peuvent y apporter -, il a la particularité de compter des spécialistes qui les étudient de façon approfondie dans une optique transculturelle : telle est la tâche des comparatistes. Au passage, il faut signaler que la terminologie juridique présente encore d'autres spécificités qui ne simplifient pas le travail du traducteur (voir p. ex. [8, p. 54]), notamment l'absence de délimitation claire avec la langue courante, l'importance de la polysémie, et un flou parfois voulu (c'est le cas dans les contextes diplomatiques).

Les problèmes causés par les divergences notionnelles entre systèmes juridiques ont suscité de nombreuses réflexions, tant chez les juristes que chez les traducteurs. Beaucoup pratiquée par les comparatistes, par exemple en France au XIX ${ }^{\mathrm{e}}$ siècle [13, pp. 100-107], la traduction peut être considérée comme un aboutissement possible de leurs recherches. Elle repose alors sur une conception exigeante [24, p. 25]. Si les principaux ouvrages généraux de droit comparé s'attardent peu sur la traduction [18, pp. 11-12], certains auteurs la traitent comme une problématique à part entière $[12 ; 32$, pp. $27-41 ; 37 ; 41$, pp. 408-413; 42]. L'évolution récente du droit européen, en particulier le projet d'élaborer un droit privé uniforme, ravive l'intérêt - et souvent l'inquiétude - des comparatistes à l'égard de la terminologie et de la traduction [24, pp. 7-19]. Elle les pousse à un questionnement philosophique sur la traductibilité du droit [18]. De leur côté, les sciences du langage ont été amenées à aborder des problématiques semblables (outre les ouvrages généraux sur le langage du droit et la traduction juridique cités en introduction, voir [30, 34, 39]). Les emprunts théoriques croisés se multiplient, de même que les efforts de synthèse adoptant un point de vue interdisciplinaire.

\subsection{Les situations de plurilinguisme officiel : une exception ?}

Dans ces réflexions qui font converger droit comparé et traduction sur le terrain de la terminologie juridique, les systèmes juridiques fonctionnant selon un régime de plurilinguisme officiel apparaissent au premier abord comme une exception. Puisque, dans ces systèmes, c'est un seul et même droit qui se dit dans plusieurs langues, les termes juridiques font théoriquement référence à des notions uniformes, définies par les sources du système en question, si bien que le droit comparé devient superflu [35, pp. 14-15]. Selon une idée répandue, il « suffit » que de vastes travaux

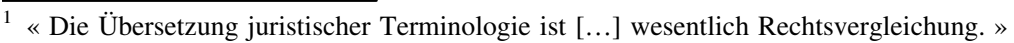


terminographiques dépouillent ces sources afin de rendre accessibles aux traducteurs les correspondances terminologiques préétablies.

Or il faut relativiser la différence de complexité entre de telles situations de traduction et celles qui exigent le passage d'un ordre juridique à un autre [39, pp. 878-879]. Principalement pour trois raisons :

1. l'existence possible de plusieurs paliers à l'intérieur d'un même ordre juridique plurilingue, du fait p. ex. d'une structure fédérale ;

2. l'interaction inévitable avec d'autres ordres juridiques, dont il faut bien pouvoir nommer les notions ;

3. la nécessité de créer des institutions nouvelles, dont la dénomination ne peut guère s'appuyer que sur l'état actuel des différentes langues en présence.

Ainsi, en Suisse, où l'organisation judiciaire relève dans une large mesure des cantons, le traducteur doit souvent passer par le droit comparé lorsqu'il a affaire à des noms de tribunaux (cas $n^{\circ} 1$ ci-dessus). Cette démarche est également nécessaire lorsqu'il faut traduire, dans le cadre du processus législatif, un rapport qui mentionne incidemment telle ou telle institution étrangère dont le législateur suisse pourrait s'inspirer (2). Le droit comparé est encore utile lorsqu'il s'agit de légiférer dans un domaine où les techniques évoluent rapidement (3). Pour illustrer ce dernier point, prenons le cas d'un projet de loi fédérale, rédigé en allemand, qui proposerait d'introduire telle ou telle nouveauté. Peut-être existe-t-elle déjà en France, en Belgique, dans l'Union européenne, au Canada... ? Le cas échéant, comment est-elle définie précisément et comment a-t-elle été dénommée en langue française ? Le traducteur doit savoir regarder au-delà des frontières pour participer à la création terminologique.

La complexité des choix terminologiques et l'utilité du droit comparé ont été illustrées à propos d'autres situations dans lesquelles un même droit s'exprime en plusieurs langues, par exemple le droit international [3] et le droit européen ([40, pp. 201-202] du point de vue de la traduction ; [29] du point de vue de l'innovation terminologique dans ce contexte dynamique et multidimensionnel). Cette problématique concerne potentiellement tous les traducteurs de textes juridiques. Elle devrait donc faire partie intégrante de leur formation quel que soit l'environnement national et régional dans lequel celle-ci se déroule.

\subsection{Une problématique à intégrer dans la formation des traducteurs}

En s'appuyant sur ces bases théoriques, il convient de définir des savoirs et des savoir-faire qui puissent trouver place parmi les objectifs d'une didactique de la traduction juridique. On peut se référer, à cet égard, au cadre élaboré par Prieto Ramos [31] (voir par ailleurs [8, pp. 37-52]). À partir des travaux consacrés aux compétences en traduction, cet auteur fait une synthèse de plusieurs modèles généraux (parmi lesquels celui du Groupe d'experts European Master's in Translation [EMT] [21]) qu'il adapte au domaine juridique. Il énumère cinq sous-compétences [31, pp. 11-14] : stratégique/méthodologique ; communicative et textuelle ; thématique et culturelle ; documentaire et technologique ; interpersonnelle et professionnelle. Dans ce cadre, l'élément «droit comparé » vient s'insérer 
principalement au niveau de la sous-compétence thématique et culturelle. Il faut toutefois relever que cet élément a aussi, de toute évidence, des liens avec d'autres dimensions : documentaire et technologique (recherche d'informations sur les systèmes juridiques dans des sources spécialisées), et surtout stratégique/ méthodologique (définition de critères d'équivalence pour le traitement des problèmes terminologiques). Cette sous-compétence primordiale, qui couvre notamment l'identification de problèmes, la mise en œuvre de procédés de traduction et la justification des décisions prises, joue un rôle clé de coordination. Elle se situe au cœur de l'activité du traducteur, dans ce que celle-ci a de plus spécifique.

Une fois précisée la place du droit comparé dans les compétences à développer, se pose la question de l'intégration concrète de cet élément dans l'enseignement. La réponse dépend dans une certaine mesure de paramètres propres à chaque formation: niveau et profil des étudiants, articulation et progression entre l'enseignement de traduction juridique et d'autres cours (traduction " générale », droit, langues spécialisées, traduction spécialisée au sens large), etc. Nous n'entrerons pas dans le détail des configurations existantes. Notre propos n'est pas non plus d'esquisser la structure interne d'un enseignement de traduction juridique (sur ce sujet, voir [6, pp. 98-101; 15, pp. 208-216]). Soulignons seulement la nécessité de jongler avec plusieurs critères de structuration : les objectifs méthodologiques, les sous-domaines du droit, les types de discours et genres textuels, les situations de traduction.

Notre réflexion vise à proposer des modes d'intégration du droit comparé qui soient adaptables à différents contextes didactiques. Elle part notamment du principe que, si les projets de traduction « en situation », empruntés à la réalité de la vie professionnelle (voir [22]), sont bien adaptés à la formation en traduction juridique [5], d'autres démarches n'en ont pas moins leur utilité. Il peut s'agir d'exposés sur le domaine de spécialité, mais aussi d'exercices ciblés permettant de «braquer le projecteur » sur tel ou tel problème de méthode présélectionné par l'enseignant (sur la complémentarité entre ce type d'approche «pédagogique » et une approche plus «professionnelle », voir [19, pp. 19-31]). S'il est essentiel de faire comprendre d'emblée que, dans la pratique de la traduction, tout entre en jeu en même temps, on peut aussi montrer l'intérêt de décomposer pour apprendre [17].

À ce stade, nous retiendrons que le droit comparé, élément méthodologique nécessaire en traduction juridique, s'intègre de façon complexe dans la compétence du traducteur, compétence qui est globalement orientée vers la prise de décisions pour résoudre des problèmes de transfert. Les sections suivantes proposent des pistes pour développer cet élément de compétence de façon ciblée, en combinant plusieurs méthodes d'enseignement.

\section{Comparer pour traduire}

\subsection{Le droit, les droits, les principes de la méthode comparative}

De quel droit comparé les traducteurs ont-ils besoin ? En d'autres termes, de quelle nature peuvent être les apports de cette discipline ? Une distinction doit être faite 
entre, d'une part, les connaissances de fond sur les systèmes juridiques et, d'autre part, les principes méthodologiques qu'il importe de savoir appliquer.

Sans un minimum de connaissances théoriques sur le phénomène juridique et sur les systèmes de droit positif correspondant à ses langues de travail, un traducteur ne peut guère entreprendre la recherche documentaire qui lui permettra, face à un problème concret, de trouver les informations qui lui manquent [15, pp. 227 et 229 ; 45, pp. 23-25]. Les sources et les structures du droit, les modes de raisonnement et les principes d'interprétation, les étapes du processus législatif, etc., constituent des grands repères à partir desquels une telle recherche est possible (sur la conception d'un cours d'introduction au droit s'adressant à des traducteurs, voir [6, pp. 88-98]). Il est souhaitable de les compléter par une initiation à quelques branches du droit [38, pp. 276-277] choisies en fonction de la combinaison linguistique. La présentation thématique d'éléments de droit comparé donne alors l'occasion d'introduire la terminologie.

Par ailleurs, les traducteurs peuvent tirer des enseignements de la réflexion théorique menée par les juristes au sujet de la méthode comparative et retenir certains principes pratiques qui en découlent [30, pp. 138-152]. Premièrement, l'équivalence absolue n'existe pas : deux institutions similaires ne sont jamais soumises à des règles rigoureusement identiques d'un système à l'autre. Ainsi, l'enfiteusi du droit italien et l'emphytéose du droit français ont en commun la plupart de leurs éléments définitoires, mais l'une peut être perpétuelle, alors que l'autre a une durée longue mais limitée. Cette différence n'est pas pertinente dans tous les contextes et n'interdit donc pas de considérer les deux notions comme équivalentes sous certaines conditions (pour d'autres exemples, voir [32, p. 41]). Deuxièmement, les comparatistes eux-mêmes considèrent que la mise en équivalence de termes juridiques ne peut être que l'aboutissement de la comparaison [41, pp. 87-118]. Qui plus est, l'étape de comparaison proprement dite ne vient qu'après un travail préliminaire visant à cerner les notions et à en faire une étude approfondie dans leur contexte juridique [30, pp. 109-115 et 140-149]. Troisièmement, la comparaison étant une opération qui tend à faire ressortir les ressemblances et les différences, elle peut fournir des critères précis pour évaluer le degré d'équivalence. Ces critères se rattachent souvent à la place de la notion dans le système (Sect. 3.2) et à sa fonction (Sect. 3.3) (pour une liste plus détaillée, voir [34, pp. 156-157]). La comparaison fournit aussi des informations pour expliquer, le cas échéant, les différences en s'appuyant sur les ressemblances, pour faire comprendre le nouveau à partir du connu.

\subsection{La notion dans le système, en synchronie et en diachronie}

Nous pourrions dire, de façon imagée, que lorsque le traducteur entre dans l'édifice du droit comparé, c'est le plus souvent à l'étage des notions et par la porte de la terminologie. Or pour le juriste, la notion n'est qu'un niveau de comparaison possible [37, pp. 228-231; 41]. On gagne souvent à situer la notion problématique dans un espace plus vaste [34, pp. 154-155], en prenant en considération d'autres niveaux : un groupe de notions (p. ex. les régimes de protection des majeurs pour comprendre la tutelle); la place de la notion par rapport aux grandes subdivisions 
du droit (procédure civile ou pénale ? compétence fédérale ou provinciale [/régionale/cantonale] ?) ; voire les grands principes sur lesquels repose l'ordre juridique. Pour illustrer ce dernier point, on peut citer le cas complexe du rapport entre Verordnung en droit allemand et règlement ou ordonnance en droit français : toute la difficulté vient ici du fait que la désignation des textes normatifs est révélatrice d'une conception différente de la séparation des pouvoirs dans le droit constitutionnel des deux pays.

Le droit comparé invite non seulement à replacer la notion dans l'ensemble du système juridique, mais aussi à prendre en considération la diversité des sources du droit [10, pp. 133-135 et 206-207]. Le concept de formant proposé par Sacco [32, pp. 43-63] aide à se représenter les effets concrets de cette diversité : pour chaque règle de droit, il existe diverses « couches »- légale, doctrinale, jurisprudentielle, etc. -, dont les rapports dépendent de la conception des sources du droit propre au système considéré. L'enseignement de la traduction juridique peut tirer de ce principe théorique une conséquence pratique quant au choix des matériaux pour l'étude des problèmes terminologiques. À un stade initial, il peut être intéressant, du point de vue pédagogique, de sélectionner des domaines dans lesquels il existe des définitions légales aisément accessibles et comparables (ex. : entre droits de la famille romano-germanique, les différentes catégories d'infractions en droit pénal ou de contrats spéciaux en droit des obligations). Il est néanmoins important de proposer ensuite un travail sur des cas de figure plus complexes (p. ex., on cherchera en vain, dans le code civil français, des règles détaillées sur les fiançailles correspondant à celles que l'on trouve dans les codes civils allemand ou italien : il faudra se tourner vers la jurisprudence).

Replacer les notions dans le contexte de chaque système afin de mieux les comprendre et les comparer, cela peut aussi signifier prendre en considération la dimension diachronique. Comment faire appréhender de façon précise à un francophone la notion de Wegfall der Geschäftsgrundlage en droit des obligations si l'on n'a pas connaissance de la situation économique à l'époque de la République de Weimar? En droit de la famille, comment traduire les termes italiens scioglimento del matrimonio et cessazione degli effetti civili del matrimonio si l'on ne sait rien de l'histoire de l'introduction du divorce en Italie ? C'est le lien entre droit et culture qui est ici en jeu. La perspective diachronique aide par ailleurs à saisir certains liens entre des notions partiellement divergentes. Ainsi, pour expliquer le rapport entre possession, Besitz et possesso, Sacco [32, pp. 35-37] propose de remonter dans l'histoire du droit pour dégager, au-delà des différences, un " génotype », une «notion superabstraite » (pouvoir + protection) dont découlent les divers «phénotypes » actuels.

Sachant qu'il peut, si nécessaire, intégrer ces différentes dimensions dans sa recherche d'équivalence, le traducteur se trouvera moins démuni. En effet, ce n'est pas parce qu'une notion n'existe pas dans la législation actuelle du système cible que le langage juridique lié à ce dernier ne possède aucun terme pour évoquer cette notion. La doctrine s'est peut-être penchée sur la question [3, p. 251]. L'histoire recèle peut-être des notions apparentées, encore présentes dans la mémoire des juristes. 


\subsection{Fonction et équivalence}

Outre la place de la notion dans le système juridique, la fonction qu'elle y remplit est le principal point d'appui pour établir des relations d'équivalence entre termes juridiques. Le recours à un critère utilitaire - plutôt qu'abstrait - pour définir de telles relations est une démarche fondamentale en droit comparé, qui a un lien évident avec les préoccupations du traducteur ([30, pp. 107-108], en se référant au traité de Zweigert et Kötz [46, pp. 33-34]). Plus précisément, il s'agit de replacer la notion dans le contexte de la règle qui $\mathrm{y}$ fait appel, et de rattacher cette règle au problème juridique qu'elle vise à résoudre [33, pp. 149-153]. Ainsi, Vanderlinden [41, pp. 50-86] propose une approche comparative de la notion de chômeur en tant qu'élément du mécanisme complexe de l'indemnisation (voir aussi pp. 193-225).

Toutefois, l'approche fonctionnelle est critiquée parmi les comparatistes car elle mettrait trop l'accent sur les ressemblances au détriment des différences, occultant l'environnement socioculturel et favorisant ainsi des équivalences approximatives. Une voie proposée par certains consiste à sortir de la comparaison directe entre deux systèmes pour élaborer d'abord des outils théoriques « neutres », métajuridiques, qui puissent servir de base [7, pp. 31-32]. Cette évolution paraît aller dans le sens d'une plus grande sensibilité à la complexité des problèmes interlinguistiques qui sont en jeu dans la traduction [24, pp. 19-21], pour autant qu'elle ne se limite pas à un discours sur le caractère irréductible des différences entre univers linguistico-culturels.

\subsection{Ressources documentaires}

Comment rassembler et synthétiser les informations nécessaires à la comparaison des notions juridiques ? Les ressources documentaires disponibles se répartissent principalement en deux catégories. Celles qui permettent de trouver séparément des informations sur chacun des systèmes en présence; et celles qui ont déjà une perspective comparative. Pour illustrer cette seconde catégorie, citons les ouvrages conçus pour présenter aux juristes d'un pays, dans leur propre langue, le droit étranger d'un autre pays avec lequel les échanges sont particulièrement intenses. Ainsi, on trouve aujourd'hui sur les rayons des bibliothèques un certain nombre de livres écrits directement en langue française à propos du droit allemand. Citons encore les ouvrages didactiques dont la publication est liée à l'internationalisation de la formation des juristes, en particulier à la mobilité des étudiants entre les facultés de droit en Europe ${ }^{2}$. Certains sites internet diffusent aussi des études de droit comparé ${ }^{3}$. Toutes ces sources de type comparatif sont très utiles sur le plan du

\footnotetext{
${ }^{2}$ Par exemple, les collections Les systèmes juridiques des États européens et Lingua juris (BadenBaden : Nomos), ou encore la collection Rechtssprache des Auslands (München/Bern : Beck/Stämpfli, en collaboration avec d'autres éditeurs européens).

3 Par exemple les sites suivants, consultés le 31 janvier 2013 : Justice - Portail - Etudes de droit comparé, http://www.justice.gouv.fr/europe-et-international-10045/etudes-de-droit-compare-10285/ ; Assemblée nationale - Comparaisons européennes, http://www.assemblee-nationale.fr/europe/comparaisons/etudes_ compares.asp ; Sénat - Europe - Les études de législation comparée, http://www.senat.fr/legislationcomparee-theme/index.html ; Juriscope - centre d'accès aux droits étrangers, http://www.juriscope.org/ les-etudes-de-droit-compare/acces-par-theme.htm.
} 
contenu, mais présentent certains dangers sur le plan de la terminologie. Nous verrons plus loin (Sect. 4.3) comment sensibiliser les (futurs) traducteurs à ce problème. Par ailleurs, si le web peut aider à se constituer des corpus sur mesure pour résoudre des problèmes précis en traduction juridique, son utilisation judicieuse suppose un apprentissage [25, pp. 244-245].

Certes, l'idéal serait de disposer de banques de données terminologiques conçues pour la traduction et intégrant l'apport du droit comparé. Les travaux déjà menés dans ce sens [20, 33, 44] trouvent aujourd'hui un prolongement dans les recherches sur les ontologies [27], mais l'ampleur du travail comparatif nécessaire en amont limite nécessairement le champ qui peut être couvert. Pour reprendre une conclusion de Mattila [23, p. 266], «there is a need for systematic study and comparison of legal institutions and concepts and their designations, from the standpoint of many languages, in defined domains ». Dans l'immédiat, les traducteurs doivent se contenter de moyens moins perfectionnés pour répondre aux exigences de précision propres au domaine juridique [24, pp. 112-113]. Une technique de travail très concrète à laquelle ils peuvent recourir pour synthétiser les résultats de leur recherche comparative est la réalisation de schémas [voir 41, pp. 87-118].

\section{De la comparaison à la traduction}

\subsection{Comparaison n'est pas traduction}

Pour le traducteur, le droit comparé n'est pas une fin en soi : traduire est une autre entreprise, à la fois plus modeste et plus ambitieuse. Il ne s'agit pas de mener une comparaison approfondie pour en exposer les résultats et, éventuellement, parvenir à des conclusions d'ordre juridique. Il s'agit de transférer dans une autre langue un message faisant appel aux notions qui ont fait l'objet de la comparaison, en produisant un texte dans une situation et pour un destinataire donnés [30, pp. 150-152]. Or dans cette situation, le but de la communication peut être de diverses natures : il ne consiste pas toujours à présenter un droit étranger en tant que tel, en le mettant explicitement en contraste avec un système cible ; la finalité peut aussi aller dans le sens d'une adaptation. Quant au destinataire, son bagage cognitif est un facteur déterminant : c'est en fonction de ce bagage que le traducteur peut concevoir un texte cible qui permette au destinataire de comprendre et d'apprendre moyennant un effort acceptable (Sandrini [34, pp. 160-163], en se fondant sur la théorie de la pertinence).

Pour nécessaire qu'il soit, le passage par le droit comparé ne peut donc pas déboucher sur des solutions toutes faites pour régler les problèmes terminologiques. Le traducteur ne doit pas en attendre des équivalences fixes qui seraient valables dans toutes les situations [39]. Si l'on part du principe que l'équivalence entre notions juridiques ne peut être que relative [43, p. 157], le droit comparé est là pour offrir une base solide permettant d'opérer des choix en fonction de critères pragmatiques [30, pp. 147-148 et 155]. 


\subsection{Une didactique axée sur le texte en situation}

Devenir un traducteur professionnel dans le domaine juridique suppose donc une prise de conscience de son rôle par rapport à celui du comparatiste. La traductologie peut contribuer à ce processus en apportant quelques principes spécifiques, qui donnent un cadre pour la pratique et encouragent à leur tour la réflexivité. En l'occurrence, ces principes concernent les types de solutions aux problèmes de divergence entre systèmes, la dimension textuelle de la traduction, et la diversité des situations communicatives et des fonctions de la traduction.

La question des types de solutions aux problèmes de divergence entre systèmes est abondamment traitée par les publications sur la traduction juridique, bien que la classification et la terminologie varient d'un auteur à l'autre (voir [12, pp. 19-35 ; 16, pp. 163-167 ; 24, pp. 85-113 ; 35, pp. 233-265] ; et le tableau présenté par Schmidt-König [37, pp. 225-226]). Schématiquement, une distinction est faite entre les emprunts, les procédés littéraux, les néologismes, les procédés fonctionnels et les procédés descriptifs (périphrase, notes du traducteur, autres ajouts paratextuels), ces différentes solutions pouvant en outre se combiner. Megale [24, pp. 99-101] fait remarquer que le concept d'équivalence fonctionnelle (voir Sect. 3.3) est courant tant en droit comparé qu'en traduction juridique. Les spécialistes des deux disciplines se rejoignent sur le principe d'un «calcul des pertes » permettant de déterminer « l'acceptabilité » de celles-ci au cas par cas [24, pp. 92-98]. À titre d'exemple, prenons la traduction d'un texte qui décrit les grandes lignes du système de publicité foncière en Allemagne. Si ce texte général mentionne de façon purement incidente, dans une énumération, une catégorie de sûreté immobilière qui n'a pas de véritable équivalent en français (p. ex. la Grundschuld ou la Rentenschuld), il est peu probable que le lecteur francophone ait besoin ici de comprendre cette notion dans toutes ses nuances : son intérêt est ailleurs. On pourra donc se contenter d'un équivalent approximatif, de type fonctionnel.

Au-delà des divers procédés d'équivalence terminologique, la comparaison des notions juridiques s'intègre dans un raisonnement portant sur le discours [43, pp. 210-211 et 215]. Or un problème de niveau terminologique peut très bien se résoudre à un niveau plus élevé, par exemple parce que toute une phrase a été reformulée [13, pp. 144-161 et 170-173]. De plus, certains choix « lourds », qui visent la précision en combinant plusieurs procédés d'équivalence, sont envisageables pour un terme qui n'a que quelques occurrences dans un texte, mais le sont moins pour un terme clé qui revient à longueur d'article. Enfin, la question se pose de la cohérence de la stratégie de traduction à l'échelle du texte dans son ensemble, certains choix de procédés pouvant être inconciliables avec d'autres. À ce stade, il est intéressant de revenir brièvement sur la question des compétences en traduction juridique (Sect. 2.3) pour souligner ceci : certes, le droit comparé relève essentiellement de la sous-compétence thématique et culturelle, mais son intégration dans le processus de traduction se fait en lien avec la sous-compétence communicative et textuelle.

Une formation en traduction juridique doit par ailleurs sensibiliser à la diversité des situations et à son influence sur la façon de traiter les problèmes terminologiques. Cette orientation fonctionnaliste a une justification d'ordre 
pratique : la variété et le rythme d'évolution des contextes professionnels demandent aux (futurs) traducteurs une grande polyvalence. Il est donc instructif pour ceux-ci de comparer des cas pratiques entre lesquels varient la nature du problème terminologique, le genre de texte ou encore la situation de traduction (Sect. 5.2.4). Voici quelques exemples des problématiques qui méritent d'être illustrées :

- le besoin, dans certaines situations, de faire reconnaître en tant que telle l'étrangeté d'une notion juridique encore inexistante dans le système cible [14] ;

- le recours volontaire à des notions vagues (bonne foi...) dont le champ d'application n'est pas nécessairement délimité de la même façon dans tous les systèmes [39, pp. 884-885] ;

- l'enchevêtrement des paliers de compétence (p. ex. fédéralisme, interactions entre les systèmes nationaux et le droit européen ou international) ;

- l'existence d'une terminologie officielle incohérente ou problématique ;

- la nécessité de créer une terminologie nouvelle.

Une vue d'ensemble de ces problématiques fournit une base solide pour adapter chaque traduction à ses destinataires. Cette démarche doit être d'autant plus consciente que l'utilisation d'outils électroniques d'aide à la traduction se généralise, avec des risques d'uniformisation excessive [4].

\subsection{Les écrits des juristes comparatistes :}

une source d'informations, et non un modèle

Après avoir insisté sur l'apport du droit comparé et sur l'utilité des écrits des comparatistes en tant que ressource documentaire, il faut-paradoxalement mettre en garde contre la reprise pure et simple de leurs choix terminologiques. En effet, il y a plus d'enseignements à tirer de leur méthode et de leurs conclusions que de leur pratique traduisante. Bestué [2] en fait la démonstration, exemples à l'appui, et rappelle que la grande différence entre le travail du comparatiste et celui du traducteur réside dans la prise en compte d'une situation de communication interculturelle. Elle conclut :

si bien es cierto que el traductor debe apoyarse en el trabajo de la doctrina y utilizar la metodología comparatista para examinar, por ejemplo, la aceptabilidad de un equivalente, consideramos que no debe olvidar que sus decisiones de traducción están marcadas, ante todo, por su particular situación comunicativa. [2, p. 210]

Cette différence de démarche est une source courante de difficultés dans l'apprentissage de la traduction juridique. La situation suivante est assez classique. Devant préparer une traduction, un étudiant a trouvé dans une revue de droit comparé un article, rédigé dans la langue cible, qui porte précisément sur le même thème que le texte source. Dans cette étude, l'une des notions posant un problème de divergence entre système source et système cible est désignée par un équivalent littéral : p. ex., dans une revue de droit comparé éditée à Paris, séparation consensuelle pour rendre le terme separazione consensuale du droit italien. 
À première vue, l'étudiant a trouvé, au terme d'une recherche documentaire sérieuse, un équivalent terminologique attesté par une source fiable et pertinente. Il importe alors de faire observer (au moyen d'un moteur de recherche) que cet équivalent se retrouve sous la plume des juristes du système juridique cible principalement lorsqu'ils parlent du système source : en l'occurrence, les juristes français n'emploient guère le terme séparation consensuelle, sauf à propos du droit italien. Il importe aussi de faire comprendre pourquoi l'étude comparative consultée par l'étudiant, bien qu'elle soit très précieuse pour comprendre l'institution étrangère en contraste avec le système cible, ne peut pas être un modèle quant aux choix terminologiques. Le comparatiste écrit dans un contexte très particulier. S'il peut faire un usage fréquent des équivalents littéraux ou des emprunts, c'est parce que tout son discours s'emploie par ailleurs à expliquer en détail la divergence des notions entre les systèmes juridiques. Le traducteur, pour sa part, travaille sur un texte qui n'est pas nécessairement centré sur cet aspect. Les emprunts ou les équivalents littéraux sont pour lui une ressource qui peut être combinée avec d'autres (des équivalents de nature explicative ou fonctionnelle) pour renforcer la précision, mais ils sont rarement autosuffisants.

\section{Types d'exercices}

\subsection{Remarques préliminaires}

Puisque l'objectif est d'apprendre à mobiliser le droit comparé pour traduire, la formation doit intégrer, à côté d'un enseignement juridique de base sous forme d'exposés, des « gros plans » méthodologiques sur certains types de problèmes de traduction mettant en jeu les divergences entre systèmes (Sect. 2.3). Ces activités doivent, d'après ce qui précède, répondre aux principes suivants : illustrer les aspects multiples de l'interaction entre traduction juridique et droit comparé ; faire ressortir la distinction entre les étapes de documentation et de comparaison (en amont) et l'étape de traduction proprement dite ; souligner l'importance de la dimension textuelle et des paramètres pragmatiques. Nous proposons ci-après une série d'exercices. Bien entendu, cette liste n'est pas exhaustive.

Nous ne détaillerons pas les modalités pratiques : p. ex. travail individuel, en petits groupes ou avec l'ensemble des participants ; réalisation pendant les heures de cours ou en dehors; type d'encadrement assuré par l'enseignant... Nous précisons toutefois que le texte source est accompagné, autant que possible, de l'énoncé d'une situation fictive (sur la formulation d'un «cahier des charges » précis en tant qu'outil pédagogique, voir [26, pp. 162-167]).

\subsection{Exemples}

\subsubsection{Prise de conscience}

- Chercher dans le système cible un texte qui soit comparable au texte source du point de vue du genre. - À un niveau global, cet exercice peut amener à prendre 
la mesure des asymétries, puisque deux systèmes juridiques distincts ne recourent pas forcément au même genre de texte pour remplir des fonctions comparables. Ainsi, dans les droits italien et français, une expropriation pour cause d'utilité publique ne s'opère pas à travers le même type de document car la décision ne relève pas du même type d'autorité.

- Traduire un extrait d'un manuel de droit qui présente en détail telle ou telle institution propre au système source, l'objectif de la traduction étant de rendre cette institution accessible à des étudiants en droit du système cible. - Cet exercice, peu réaliste par rapport à la pratique professionnelle, a un intérêt pédagogique en début de formation: il demande un travail comparatif approfondi sur une notion ou un groupe de notions.

\subsubsection{Identification}

- Identifier dans un texte source les termes nécessitant un passage par le droit comparé. - Cet exercice consiste en un premier balisage qui prépare une recherche approfondie.

- Identifier à partir d'un texte source un groupe de notions connexes ; grâce à une recherche documentaire, esquisser le réseau notionnel qui lui correspondrait dans le système juridique cible.

\subsubsection{Recherche et comparaison}

- Constituer un dossier documentaire, éventuellement en petit groupe, en veillant à la traçabilité des informations.

- Récapituler les résultats d'une recherche comparative dans un tableau ou un schéma.

\subsubsection{Choix et justification}

- À propos d'un problème terminologique donné, énumérer des équivalents possibles hors contexte et indiquer ceux qui sont envisageables en contexte ; analyser ces solutions en appliquant la typologie des procédés présentée préalablement (voir ici Sect. 4.2) ; justifier la solution choisie.

- Rédiger une note du traducteur pour compenser une divergence entre notions.

- Comparer le traitement de plusieurs problèmes terminologiques illustrant des cas de figure différents. - L'analyse cherchera à expliciter le lien entre la nature de chaque problème et la solution apportée.

- Comparer le traitement du même problème terminologique dans des genres de textes différents, ou encore dans le même texte mais dans des situations différentes (multiciblage).

- Après avoir réalisé une traduction dans des conditions professionnelles (p. ex. lors d'un stage ou d'une simulation), commenter spécifiquement les difficultés 
liées aux divergences entre les notions, en mettant en relief la cohérence de la stratégie adoptée à cet égard.

\subsubsection{Observation et critique}

- Commenter (et éventuellement comparer) des exemples historiques de traductions dans le domaine de droit : catégories d'équivalents employés dans les cas de divergence notionnelle, catégories d'éléments paratextuels, catégories d'arguments utilisés dans le paratexte pour justifier les choix de traduction. - Cet exercice peut donner l'occasion d'analyser des cas « atypiques », p. ex. la résolution indirecte d'un problème terminologique par la reformulation globale d'une phrase.

- Analyser les pratiques de certaines institutions (p. ex. types d'équivalents privilégiés) face aux problèmes découlant des divergences entre systèmes juridiques. - Voir [25, pp. 243-244] à propos de l'exploitation des corpus bilingues parallèles dans l'enseignement de la traduction juridique.

\subsubsection{Théorisation}

- Lire, présenter et commenter des études théoriques portant sur les questions de droit comparé en traduction juridique.

\section{Conclusion}

Comment apprendre à comparer les droits pour traduire les textes juridiques ? En réponse à cette question, le présent article propose un cadre didactique de type fonctionnaliste : il s'agit de passer en revue les interactions du droit comparé avec le processus de traduction, à travers la confrontation de divers cas de figure et situations. Notre réflexion s'est concentrée sur la résolution des problèmes terminologiques et sur le développement de cette compétence grâce à des activités didactiques ciblées.

La mondialisation fait aujourd'hui du plurilinguisme un facteur important dans la transformation des langues spécialisées, et notamment du langage du droit. La formation des traducteurs doit les rendre capable de participer, en collaboration avec d'autres acteurs [22, pp. 13-14], à ce processus dans lequel la terminologie joue un rôle de premier plan. L'évolution des profils professionnels conduira peut-être à inverser les termes de notre problématique pour adopter la perspective de l'enseignement du droit : du «droit comparé pour traducteurs » à la «traduction pour juristes comparatistes », de « savoir comparer les droits pour traduire » à « savoir traduire pour faire du droit comparé ». C'est du moins l'une des pistes que suggère le récent livre de Ost [28, pp. 379-417].

Du côté de la didactique de la traduction, la démarche suivie dans le présent article pourrait être appliquée à d'autres éléments de compétence. La résolution des problèmes relevant du discours juridique et de ses conventions est, en particulier, un champ qui se prêterait à la conception d'exercices à partir d'une synthèse 
interdisciplinaire des réflexions théoriques. Le but est de proposer, en matière de traduction juridique, une formation cohérente articulée autour des compétences propres à cette activité.

\section{Références}

1. Arntz, Reiner. 2010. Juristisches Übersetzen zwischen Sprachvergleich und Rechtsvergleich. Lebende Sprachen 55(1) : 17-30.

2. Bestué, Carmen. 2008. El método comparativo en la traducción de textos jurídicos: úsese con precaución. Sendebar 19 : 199-212.

3. Bestué, Carmen. 2009. La traducción de términos jurídicos en el ámbito internacional. Babel 55(3) : 244-263.

4. Bestué, Carmen, et Mariana Orozco. 2011. La necesidad de la naturalidad en la reformulación en la traducción jurídica en la "era de la automatización" de las traducciones. The Journal of Specialised Translation 15 : 180-199. http://www.jostrans.org/issue15/art_bestue.pdf. Consulté le 31 janvier 2013.

5. Biel, Łucja. 2011. Professional realism in the legal translation classroom: Translation competence and translator competence. Meta : Journal des Traducteurs 56(1) : 162-178.

6. Bocquet, Claude. 2008. La traduction juridique : Fondement et méthode. Bruxelles : De Boeck.

7. Brand, Oliver. 2009. Language as a barrier to comparative law. In Translation issues in language and law, dir. Frances Olsen, Alexander Lorz et al., 18-34. New York : Palgrave Macmillan.

8. Cao, Deborah. 2007. Translating law. Clevedon/Buffalo : Multilingual Matters.

9. Colina, Sonia. 2003. Translation teaching: From research to the classroom: A handbook for teachers. Boston : McGraw-Hill.

10. Constantinesco, Léontin-Jean. 1974. Traité de droit comparé, vol. 2. Paris : LGDJ.

11. De Groot, Gerard-René. 1991. Recht, Rechtssprache und Rechtssystem. Betrachtungen über die Problematik der Übersetzung juristischer Texte. Terminologie et Traduction 3 : 279-316.

12. De Groot, Gerard-René. 1999. Das Übersetzen juristischer Terminologie. In Recht und Übersetzen, dir. Gerard-René De Groot et Reiner Schulze, 11-46. Baden-Baden : Nomos.

13. Dullion, Valérie. 2007. Traduire les lois : un éclairage culturel. La traduction en français des codes civils allemand et suisse autour de 1900. Cortil-Wodon : E.M.E.

14. Ferran Larraz, Elena. 2009. La institución desconocida y la intraducibilidad. Paralelismo entre el derecho comparado y la traducción juridical frente a la intraducibilidad. Meta: Journal des Traducteurs 54(2) : 295-308.

15. Garzone, Giuliana. 2007. Osservazioni sulla didattica della traduzione giuridica. In Tradurre le microlingue scientifico-professionali: Riflessioni teoriche e proposte didattiche, dir. Patrizia Mazzotta et Laura Salmon, 194-238. Torino : UTET.

16. Gémar, Jean-Claude. 1995. Traduire ou l'art d'interpréter, vol. 2. Québec : Presses de l'Université du Québec.

17. Gile, Daniel. 1995. Basic concepts and models for interpreter and translator training. Amsterdam/ Philadelphia : Benjamins.

18. Glanert, Simone. 2011. De la traductibilité du droit. Paris : Dalloz.

19. González Davies, Maria. 2004. Multiple voices in the translation classroom: Activities, tasks and projects. Amsterdam/Philadelphia : Benjamins.

20. Grass, Thierry. 1999. La traduction juridique bilingue français-allemand: Problématique et résolution des ambiguités terminologiques. Bonn : Romanistischer Verlag.

21. Groupe d'experts EMT. 2009. Compétences pour les traducteurs professionnels, experts en communication multilingue et multimédia. Bruxelles. http://ec.europa.eu/dgs/translation/programmes/ emt/key_documents/emt_competences_translators_fr.pdf. Consulté le 31 janvier 2013.

22. Kiraly, Don. 2000. A social constructivist approach to translator education: Empowerment from theory to practice. Manchester : St. Jerome.

23. Mattila, Heikki E.S. 2006. Comparative legal linguistics. Traduit par Christopher Goddard. Aldershot : Ashgate. 
24. Megale, Fabrizio. 2008. Teorie della traduzione giuridica. Fra diritto comparato e "translation studies". Napoli : Editoriale Scientifica.

25. Monzó Nebot, Esther. 2008. Corpus-based activities in legal translator training. The Interpreter and Translator Trainer 2(2) : 221-252.

26. Nord, Christiane. 1995 [1988]. Textanalyse und Übersetzen: Theoretische Grundlagen, Methode und didaktische Anwendung einer übersetzungsrelevanten Textanalyse. $3^{\mathrm{e}}$ éd. Heidelberg : Groos.

27. Orozco, Mariana, et Pilar Sánchez-Gijón. 2011. New resources for legal translators. Perspectives: Studies in Translatology 19(1) : 25-44.

28. Ost, François. 2009. Traduire : Défense et illustration du multilinguisme. Paris : Fayard.

29. Peruzzo, Katia. 2012. Secondary term formation within the EU: Term transfer, legal transplant or approximation of Member States' legal systems? The Journal of Specialised Translation 18 : 175-186. http://www.jostrans.org/issue18/art_peruzzo.pdf. Consulté le 31 janvier 2013.

30. Pommer, Sieglinde. 2006. Rechtsübersetzung und Rechtsvergleichung. Translatologische Fragen zur Interdisziplinarität. Frankfurt a.M. : Lang.

31. Prieto Ramos, Fernando. 2011. Developing legal translation competence: An integrative processoriented approach. Comparative Legilinguistics International Journal for Legal Communication 5 : $7-21$.

32. Sacco, Rodolfo. 1992 [1979]. Introduzione al diritto comparato. $5^{\mathrm{e}}$ éd. Torino : UTET.

33. Sandrini, Peter. 1996. Terminologiearbeit im Recht. Deskriptiver begriffsorientierter Ansatz vom Standpunkt des Übersetzers. Wien : TermNet.

34. Sandrini, Peter. 2009. Der transkulturelle Vergleich von Rechtsbegriffen. In Legal language in action: Translation, terminology, drafting and procedural issues, dir. Susan Šarčević, 151-165. Zagreb : Nakladni zavod Globus.

35. Šarčević, Susan. 1997. New approach to legal translation. The Hague/London/Boston : Kluwer Law International.

36. Scarpa, Federica. 2008 [2001]. La traduzione specializzata. Un approccio didattico professionale. $2^{\mathrm{e}}$ éd. Milano : Hoepli.

37. Schmidt-König, Christine. 2005. Die Problematik der Übersetzung juristischer Terminologie. Eine systematische Darstellung am Beispiel der deutschen und französischen Rechtssprache. Münster : LIT.

38. Sparer, Michel. 2002. Peut-on faire de la traduction juridique ? Comment doit-on l'enseigner ? Meta : Journal des Traducteurs 47(2) : 265-278.

39. Terral, Florence. 2004. L'empreinte culturelle des termes juridiques. Meta : Journal des Traducteurs 49(4) : 876-890.

40. Tranchant, Isabelle. 2011. Les méthodes de traduction et la terminologie juridique : L'expérience du département de langue française de la Commission européenne. In Traduction du droit et droit de la traduction, dir. Xavier North, 199-206. Paris : Dalloz.

41. Vanderlinden, Jacques. 1995. Comparer les droits. Diegem : Kluwer/Story Scientia.

42. Weisflog, Walter E. 1996. Rechtsvergleichung und juristische Übersetzung. Eine interdisziplinäre Studie. Zürich : Schulthess.

43. Wiesmann, Eva. 2002. La traduzione giuridica dal punto di vista didattico. In Traduttori e giuristi a confronto: Interpretazione traducente e comparazione del discorso giuridico, dir. Leandro Schena et Rita D. Snel Trampus, vol. 2, 205-217. Bologna : CLUEB.

44. Wiesmann, Eva. 2004. Rechtsübersetzung und Hilfsmittel zur Translation. Wissenschaftliche Grundlagen und computergestützte Umsetzung eines lexikographischen Konzepts. Tübingen : Narr.

45. Wiesmann, Eva. 2009. Rechtstexte - Eine übersetzerische Herausforderung und ihre didaktischen Konsequenzen. In Eindeutig uneindeutig: Fachsprachen - ihre Übersetzung, ihre Didaktik, dir. Piergiulio Taino, Marina Brambilla et al., 11-26. Frankfurt a.M. : Lang.

46. Zweigert, Konrad, et Hein Kötz. 1996 [1969]. Einführung in die Rechtsvergleichung auf dem Gebiete des Privatrechts. $3^{\mathrm{e}}$ éd. Tübingen : Mohr. 